\title{
TESTING AND ASSEMBLY OF WIMS CUBES CONTAINING PASSIVE AND ACTIVE INTEGRATED CABLES
}

\author{
Asli B. Ucok, Joseph M. Giachino, and Khalil Najafi \\ Center for Wireless Integrated Microsystems (WIMS) \\ University of Michigan, Ann Arbor, MI 48109-2101
}

\begin{abstract}
This paper presents the first assembly and electrical testing results of a packaging/assembly approach for wireless integrated microsystems (the WIMS Cube). In this assembly approach, the microsystem dice are placed inside a cube. Electrical/fluidic signal transfer between dice can be achieved in one of two ways. The first method uses rows of integrated active flexible cables that are attached to the cube walls and actuated during assembly, row-byrow, to connect to the dice. The second method uses passive flexible cables integrated with separate connector dice that are interleaved between device dice inside the cube. In both methods, flexible cables make pressure contacts to the pads and fluidic inlets of each microsystem die, thus forming a mechanical connect/disconnect system for both electrical and fluidic signals. WIMS Cube prototypes that are $5 \mathrm{~mm}$ and $10 \mathrm{~mm}$ on a side were fabricated. Cubes with integrated Parylene cables were tested for reliable pressure-contact electrical connections. A low contact resistance of $4.2 \Omega$ was achieved between cables and dice. Reliability was demonstrated by electrically connecting and disconnecting the passive flexible cables $\sim 10$ times. Fluidic connector cables that can eventually interface with fluidic substrates were integrated with the Cube.
\end{abstract}

\section{INTRODUCTION}

Today's packages for integrated circuits and microsystems should protect them from the external environment and allow electrical connectivity without affecting performance. Current packaging and assembly approaches do not provide the flexibility and modularity needed for a microsystem containing substrates with sensors, actuators, and circuits fabricated using different technologies. We introduced the concept of a packaging/assembly

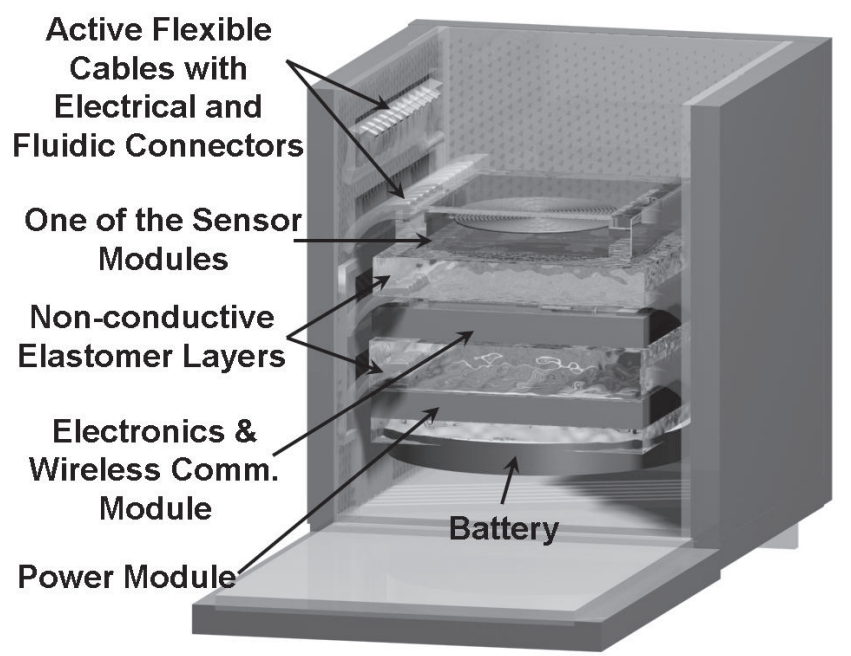

Figure 1. WIMS Cube with active flexible cables: The flexible cables are actuated into position using on-chip actuators and make pressure contacts to pads and fluidic inlets on the dice. approach for wireless integrated microsystems (the WIMS Cube) [1], whereby multiple substrates containing circuits, sensors/actuators or instruments are stacked on top of one another in a re-workable and modular fashion inside a Cube, with interconnection between substrates provided through Cube walls and flexible cables $[\mathbf{2 , 3}]$. The WIMS Cube concept represents a significant departure from standard 3D chip assembly and packaging approaches $[\mathbf{4 , 5}]$ in that it makes the assembly and connection reworkable, thus providing maximum flexibility when assembling/reassembling and packaging complex microsystems. In this paper, we report the first assembly and electrical testing results of a complete WIMS Cube containing multiple test substrates, the development of WIMS Cubes utilizing only "passive" cables that do not require actuation, and the successful fabrication and integration of fluidic cables that can eventually interface with fluidic substrates.

\section{WIMS CUBE STRUCTURE AND OPERATION}

Figures 1-2 illustrate WIMS Cubes with active and passive cables, respectively. The Cube can be as small as a few millimeters or as large as a few centimeters on a side and holds different dice. Electrical/fluidic signals are transferred between dice using either rows of integrated flexible cables attached to the Cube walls and actuated, row-by-row, to make pressure contacts to each die (Figure 1), or by passive cables integrated on connector dice that are interleaved between device dice (Figure 2). In case of active cables, all cables are retracted at start of assembly. After each die is "dropped" into the Cube, a row of cables is actuated/released into position. A non-conductive elastomer layer is dropped over the die, followed by another die; the process is repeated. In case of passive cables, the connector dice containing

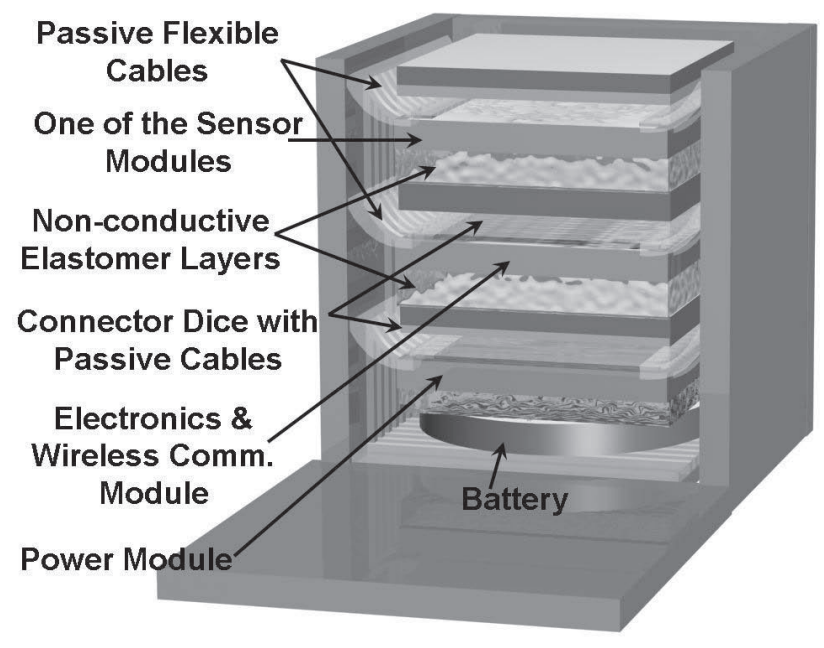

Figure 2. WIMS Cube with passive flexible cables: WIMS Cube has connector dice with integrated passive flexible cables that make pressure contacts to system dice and cube sidewall, where routing of all signals is achieved. 
connection pads press onto their corresponding device dice to make connections. The signal is then transferred through the passive cables, which extrude at the edge of each connector die and connect to the Cube sidewall; in this case the passive cables are bent as the connector dice are dropped into the Cube. A nonconductive elastomer layer is used as a cushion between dice to accommodate local variations in pressure/distance. In both approaches, the Cube is covered by a lid, which presses against the dice and elastomers, forcing all cables into intimate contact with their substrate.

The pressure-contact connections are not permanent. This allows the microsystem to be disassembled either if one of the dice fails after assembly, or if a different microsystem with a different set of sensors or actuators is needed for another application, resulting in maximum flexibility and modularity, two critical features for microsystem applications.

\section{ASSEMBLY OF THE WIMS CUBE}

The WIMS Cube is fabricated using planar silicon technology. The bottom and the four sidewalls of the Cube are fabricated on a single substrate and are attached to each other by the flexible cable material, and a separate piece could be used as the lid of the cube. Each cube can then be handled easily and folded using a jig. The Cube sidewalls are fabricated so that the sidewalls and Cube base can be brought together like a jigsaw puzzle for good alignment as shown in Figure 3, and then glued/soldered to form the Cube.

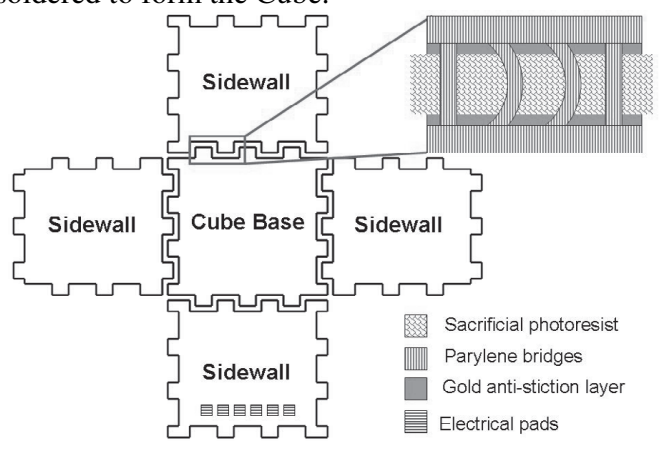

Figure 3. Layout of the WIMS Cube

Figure 3 shows the sidewall-to-base connector bridges that carry all the metal routing for both the actuation of each row of cables as well as the electrical connection layer. Careful handling of the Cube is necessary as these bridges may be torn, disconnecting the access to the connectors on a sidewall. These bridges should be flexible as well as rugged, and with a careful design, the probability of tear can be minimized. The bridges may be designed as multiple narrow connectors instead of a wide Parylene bridge to increase their flexibility.

Assembly platforms and a multi-tip vacuum pick for the assembly and handling of the fabricated package were also developed to improve the reliability of the handling and assembly process. The assembly platform is a jig with vacuum connections to hold the sidewalls of the Cube before it is permanently brought together, and to populate the Cube with test dice for connection tests. The jig is fabricated using silicon micromachining techniques. The assembly platform with latches and polymer hinges, shown in Figure 4, was designed to enable Cube assembly with less handling and better yield. This platform is assembled together with the Cube itself, and the latches lock the sidewalls in place during assembly.

Parylene is a very rugged material, and does not tear apart easily. However, due to bending and twisting, one can still damage the sidewall-to-base connector bridges. As a result, a special multi-tip vacuum pick was designed in order to help in handling the WIMS Cubes (Figure 5).

The following section on fabrication will present the fabrication of the WIMS Cube as well as the assembly platform and the multi-tip vacuum pick.

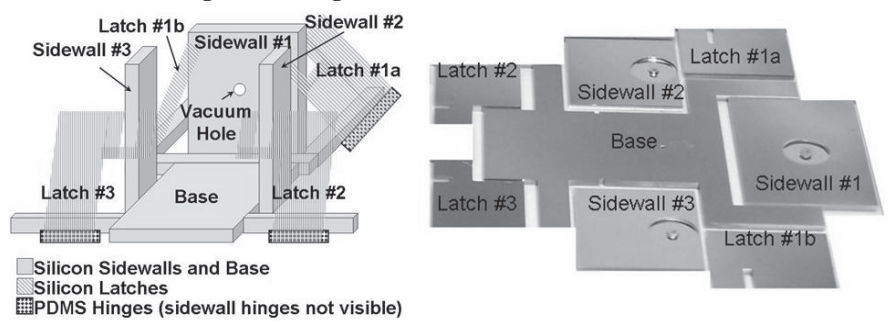

Figure 4. Schematic of the assembly platform with latches and polymer hinges, and a fabricated assembly platform

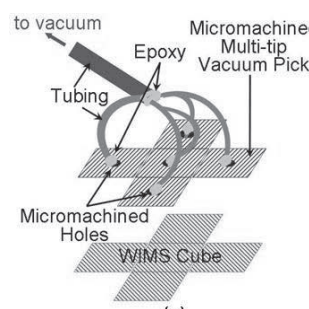

(a)

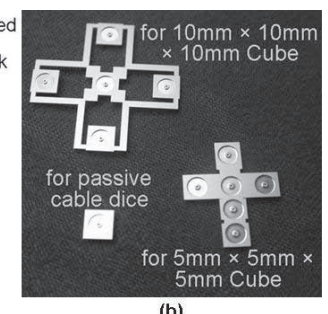

(b)

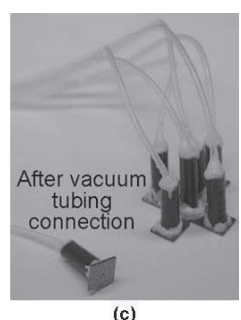

(c)
Figure 5. (a) Schematic of the multi-tip vacuum pick, (b) various vacuum picks after fabrication, and (c) vacuum picks after the tubing connection

\section{FABRICATION}

Figure 6 shows the 12-mask surface micromachining process for WIMS Cubes with active or passive cables with integrated electrical and fluidic connectors. The bottom cross-sectional view on the right in Figure 6 shows a connector die with electrical and fluidic connectors fabricated using the same process used for Cubes with active cables. The layers that exist for active cables, but not for passive cables, are merely removed from passive cables. Parylene is the material of choice for the cables of the WIMS Cube, since it is quite flexible, and provides sufficient chemical inertness, mechanical strength and stability $[2,3]$.

WIMS Cube prototypes that are $5 \mathrm{~mm}$ and $10 \mathrm{~mm}$ on a side were fabricated. Fabrication starts with an oxidized wafer (Figure 6-a). This oxide layer is used for the insulation of pads used for actuation of rows of cables and testing the electrical connectors during cube assembly. It does not need to be too thick as long as it provides good insulation. A $3500 \AA$-thick oxide layer is thermally grown and patterned for this process. A $\mathrm{Cr} / \mathrm{Au}$ layer of $500 \AA / 1500 \AA$ is deposited and patterned as an anti-stiction layer underneath connector bridges. Gold is a hydrophobic layer, and the probability of Parylene to stick to gold is less compared to sticking to silicon, silicon dioxide, and Parylene itself. Patterning of a sacrificial photoresist layer $(1.3-1.5 \mu \mathrm{m})$ is followed by the deposition and patterning of the first Parylene layer $(\sim 2 \mu \mathrm{m})$. Parylene is dry-etched in $\mathrm{O}_{2}$ plasma. The electrode metal layer (5000^-thick gold layer), which forms the coils for thermomagnetically actuated cables [3], is deposited and patterned. Although released Parylene structures do not stick to gold surfaces in a wet release process, gold film directly deposited on Parylene is found to adhere well as opposed to its adhesion problem to silicon. The soft gold layer covers the slightly rough Parylene surface well, resulting in good adhesion. By using gold alone, patterns as small as $7 \mu \mathrm{m}$ can easily be patterned. A hard-baked photoresist layer ( $4 \mu \mathrm{m}$ after hard-bake) at the tips of the electrical connectors on 


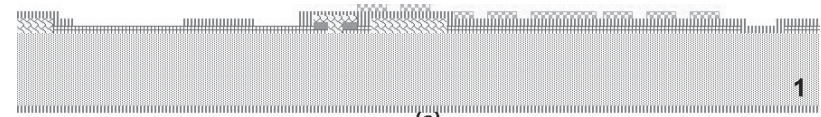

(a)

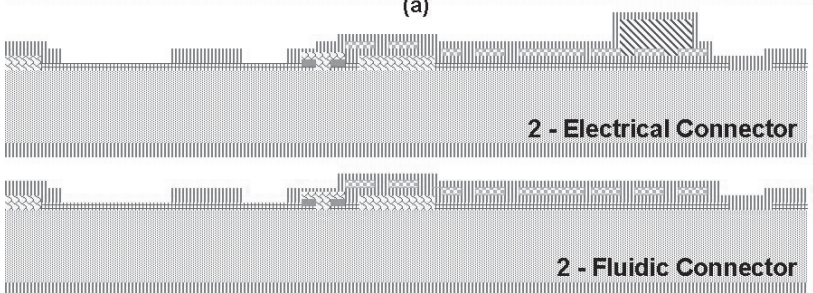

(b)
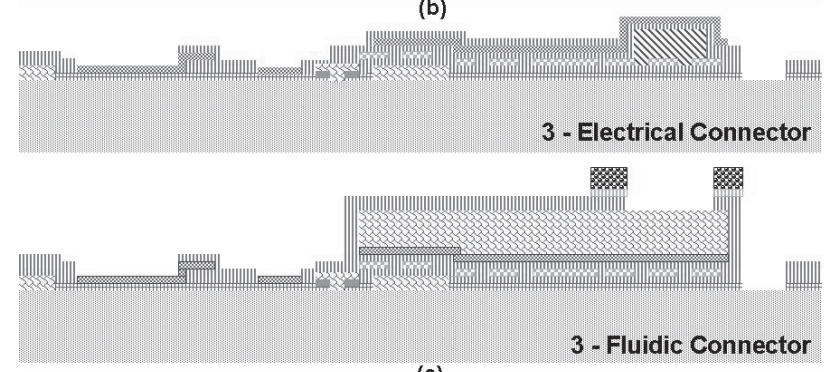

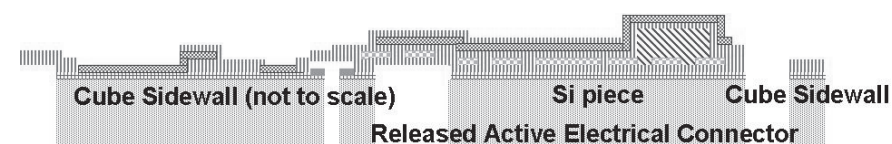

Released Active Electrical Connector
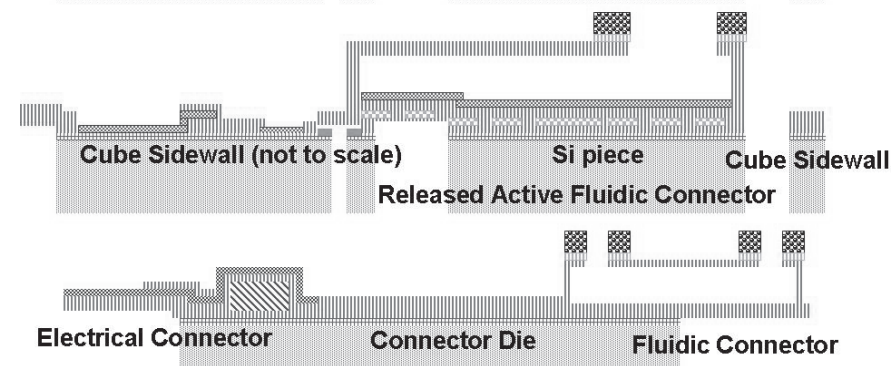

Released Connector Die with Passive Cables

(d)

$\begin{array}{lll}\text { Si Substrate } & \text { (d) } & \text { Photoresist }\end{array}$

Au Anti-stiction Layer Electrode Metal |||||| Ti mask

1. SU-8

Figure 6. Process flow of the WIMS Cubes with integrated active or passive cables with electrical and fluidic connectors

both active and passive flexible cables forms extruding tips for pressure-contact electrical connections (Figure 6-b). Next, the second Parylene layer is deposited and patterned to open contacts on the coils. A Cr/Au layer of thickness $250 \AA / 5000 \AA$, is deposited to form the electrical interconnects of the cables, and the antistiction/anti-collapse bottom layer for the fluidic channels (Figure 6-c). The material and thickness for this metal layer is affected by many factors including the stress-compensation of the thin-film stack and the formation of low electrical routing resistances for the Cube. The sacrificial photoresist layer for the fluidic channels is then patterned. The thickness of the photoresist determines the height of the channels, and thicknesses of $8-12 \mu \mathrm{m}$ were used for this process. It is followed by the deposition of the final Parylene layer $(\sim 2 \mu \mathrm{m})$. A $3000 \AA$-thick Ti layer is sputtered and patterned as a mask for the final Parylene etch. The Ti layer should be sputtered and be thick enough to make sure the large step height of the fluidic channels is covered well for the final Parylene etch. Ti layers thicker than $3000 \AA$ delaminate at sharp corners, like the corners of the cables, after patterning. Ti is chosen as the mask layer, because it adheres to Parylene well, and the Ti etchant $\left(\mathrm{NH}_{4} \mathrm{OH}: \mathrm{H}_{2} \mathrm{O}_{2}: \mathrm{H}_{2} \mathrm{O}=1: 1: 4\right)$ does not damage the thin film stack of the cables during Ti mask patterning and removal. Next, a $13 \mu \mathrm{m}$-thick SU-8 layer, which is used for forming o-ring structures at the inlets of fluidic connectors, is patterned. After etching the Parylene in order to obtain the cable structures, the Ti mask is stripped. Lastly, a backside through-wafer etch is performed to separate the Cube walls, form active flexible cables and help release passive flexible cables (Figure 6-d). WIMS Cubes are then released by removing the sacrificial photoresist layer. A novel ultrasonic agitation release/dry technique is used that combines the advantages of the gold hydrophobic layer as an anti-stiction layer and the energy provided by ultrasonic agitation.

The assembly platforms and the vacuum pick are micromachined using a 2-mask DRIE etch process for forming the cavities for vacuum tubing. The jig is one piece with PDMS hinges. The tubing is attached using epoxy.

Figures 7-8 show Cubes during assembly and testing using two different micromachined jigs. As shown in Fig. 8, Cubes with multiple test substrates containing bonding pads and interconnects have been assembled. The tests were performed using a probe station. After the three sidewalls of the Cube are folded and secured by the vacuum connections of the assembly platform, probe tips are used to access the electrical pads on the unfolded sidewall of the Cube. The test chips and non-conductive elastomer layers populating a WIMS Cube with passive flexible cables can be seen in the inset of Figure 8.

Figure 9 shows various cables after release. The silicon piece underneath the active flexible cables creates firm pressure-contact connections to the system dice during the Cube assembly. Passive flexible cables have finger-like tips for better and more reliable connection with the metal lines on the Cube sidewall.

\section{ELECTRICAL TESTING}

The fabricated WIMS Cubes with integrated Parylene cables were tested for reliable pressure-contact electrical connections. For the WIMS Cubes with passive flexible cables, the total resistance of the electrical connection system that consists of two passive cables is measured. Each of these cables makes one pressure contact to a pad on a test die and another pressure contact to the Cube sidewall. The measured resistance included the negligible resistance of the metal line between the pads (calculated to be $0.07 \Omega$ ) on a test die, as well as the four pressure-contact resistances, and the resistances of the two passive flexible cables. Due to the duality of the measured components, half of the measured resistance is graphed in Figure 10 as the electrical resistance per passive electrical connector with two pressurecontact connections. A low contact resistance of $4.2 \Omega$ was achieved between cables and dice. Reliability was demonstrated by electrically connecting and disconnecting the passive flexible cables $\sim 10$ times. The variation in each measurement of Figure 10 depends on the force applied on the stack; the higher the applied force, the lower the pressure-contact resistance.

For WIMS Cubes with active cables, the Cube is designed so that four-point probe measurement of two pressure-contact connections with a negligible metal line resistance of the test dice included can be measured. Smaller than $1.9 \Omega$ resistance per two pressure-contact connections was achieved by testing active flexible cables. These cables were not actuated using the on-chip actuators but were manually pushed into position. Tests on fluidic connectors have not been performed. 


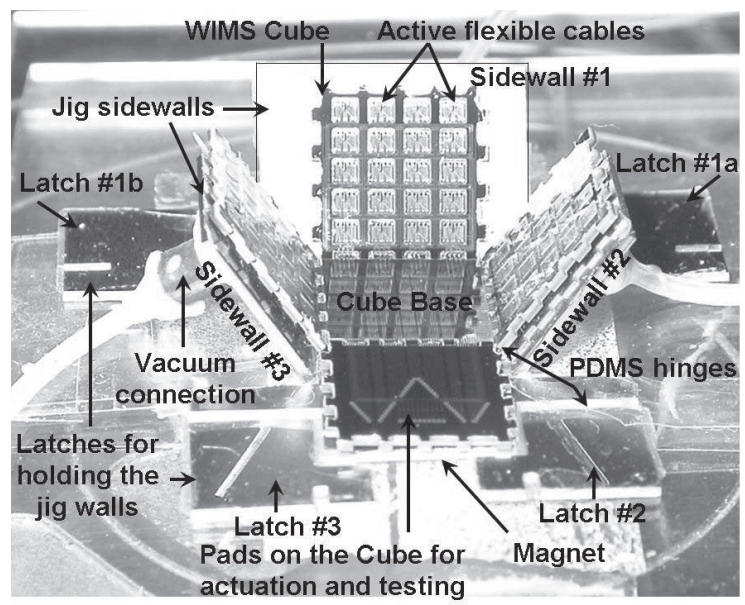

Figure 7. A WIMS Cube with active cables during assembly. The platform is a jig with latches and PDMS hinges.

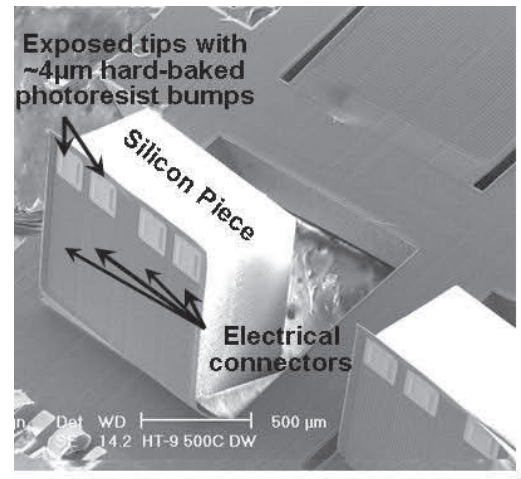

(a)

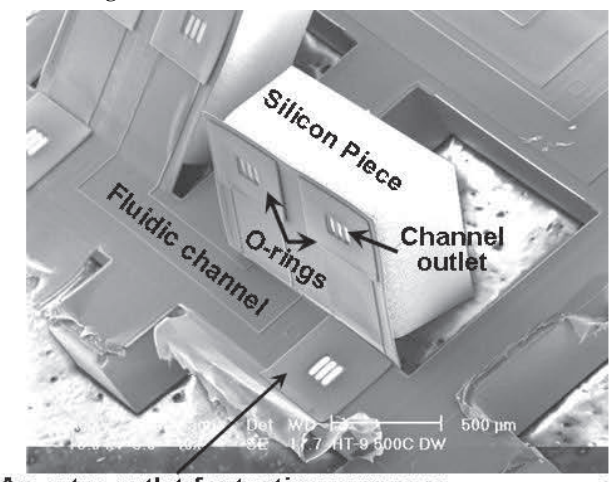

(b)

An extra outlet for testing purposes

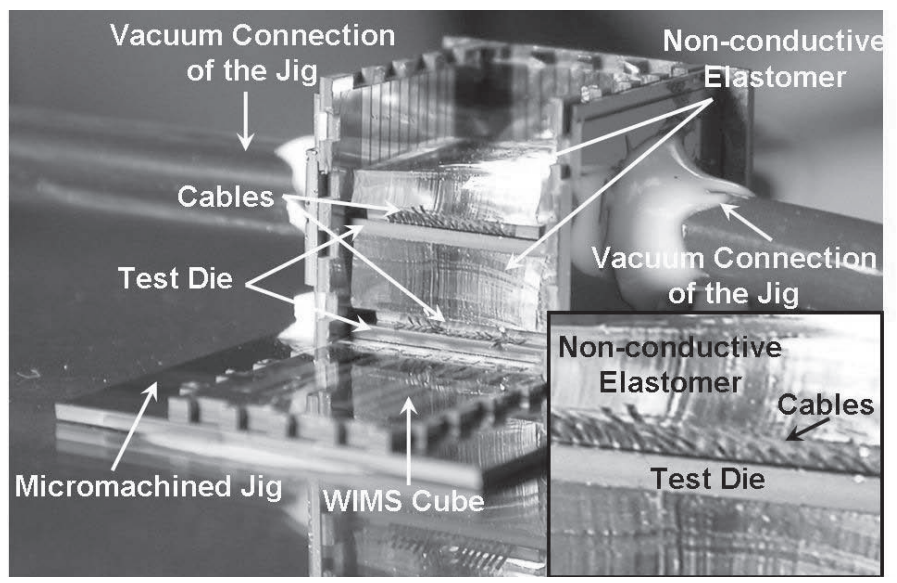

Figure 8. A WIMS Cube with passive cables during testing. The platform is a permanently assembled jig. The Cube is dropped into the jig using a flat platform not shown in the picture.

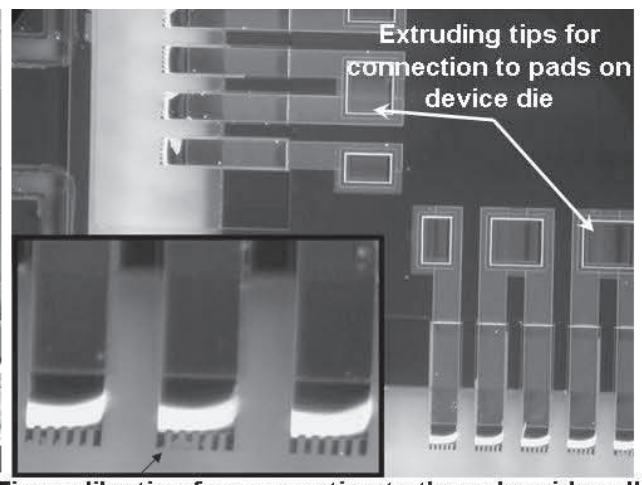

(c)

Figure 9. Released active flexible cables (a) with four electrical connectors and (b) with fluidic connectors, and (c) Released passive electrical connectors with finger-like tips for better contact to the metal lines on the Cube sidewall

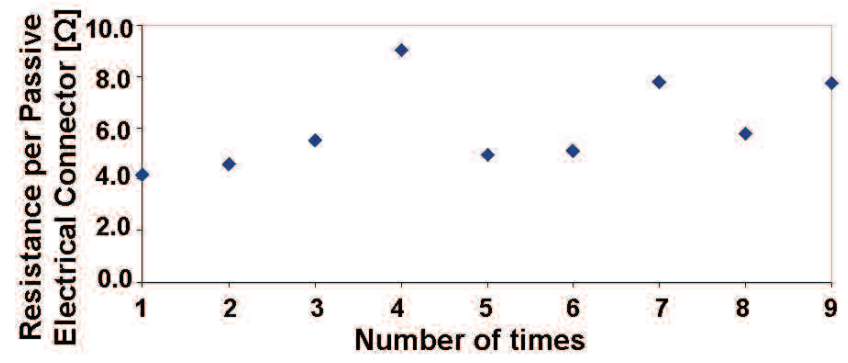

Figure 10. Measured electrical resistance per passive electrical connector. Each passive electrical connector makes two pressure-contact connections; one to the pad on the system die and the other to the Cube sidewall.

\section{CONCLUSIONS}

We reported the first assembly and electrical testing results of a complete WIMS Cube containing multiple test substrates, the development of WIMS Cubes utilizing only "passive" cables that do not require actuation, and the successful fabrication and integration of fluidic cables that can eventually interface with fluidic substrates. Repeated measurements of pressure-contact electrical resistance for passive cables as low as $4.2 \Omega$ were achieved. These resistances are repeatable after tens of connectdisconnect experiments (a Cube is envisioned not to undergo more than a few tens of reworking sessions). Tests on fluidic connectors have not been performed yet.

\section{ACKNOWLEDGMENTS}

The authors thank Brendan E. Casey for helpful discussions on the assembly setup. This work was supported by the Engineering Research Centers Program of the National Science Foundation under Award Number EEC-9986866.

\section{REFERENCES}

[1] A.B. Ucok, J.M. Giachino, K. Najafi, "Compact, Modular Assembly and Packaging of Multi-Substrate Microsystems," Tech. Digest, $12^{\text {th }}$ Int. Conf. Solid-State Sensors, Actuators, and Microsystems, Boston, MA, 2, 2003, pp.1877-1878.

[2] A.B. Ucok, J.M. Giachino, K. Najafi, "The WIMS Cube: A Microsystem Package with Actuated Flexible Connections and Re-workable Assembly," Tech. Digest 2004 Solid-State Sensor and Actuator Workshop, Hilton Head, SC, 2004, pp.117-120.

[3] A.B. Ucok, J.M. Giachino, K. Najafi, "Modular Assembly/Packaging of Multi-Substrate Microsystems (WIMS Cube) Using Thermo-Magnetically Actuated Cables," Tech. Digest, $18^{\text {th }}$ IEEE Int. Conf. Micro Electro Mechanical Systems, Miami, FL, 2005, pp. 536-539.

[4] H. Goldstein, "Packages Go Vertical," IEEE Spectrum, 38, No. 8, 2001, pp. 46-51.

[5] B. Murari, "Bridging the Gap between the Digital and Real Worlds: The Expanding Role of Analog Interface Technologies," Tech. Digest, IEEE Int. Solid-State Circuits Conf., 1, 2003, pp. 30-35. 